\title{
Perspektivenerweiterung durch kritische Gender Studies: Gendersensible Bildung für nachhaltige Entwicklung im GW-Unterricht
}

\author{
petra.wlasak@uni-graz.at, Regionales Zentrum für Expertise für Bildung für nachhaltige Entwicklung Graz-Styria, Karl-Franzens-Uni- \\ versität Graz \\ "*julia.wlasak@uni-graz.at, Regionales Zentrum für Expertise für Bildung für nachhaltige Entwicklung Graz-Styria, Karl-Franzens-Uni- \\ versität Graz
}

eingereicht am: 30.05.2017, akzeptiert am: 23.10.2017

In den didaktischen Grundsätzen und Basiskonzepten des GW-Unterrichts sind die Prinzipien von Bildung für nachhaltige Entwicklung (BNE) verankert. BNE hat das Ziel, Lernende zu befähigen, sich an der Gestaltung einer nachhaltigen und gerechten Zukunft für alle zu beteiligen. Trotz dieses holistischen und partizipativen Ansatzes von BNE fehlt bis dato ein Bewusstsein für eine gendersensible Umsetzung von BNE, die bestehende Geschlechterungleichheiten ins Blickfeld nimmt. Stattdessen dominieren essentialistische, viktimisierende oder geschlechtsblinde Diskurse. Im Sinne des Gerechtigkeitsansatzes von BNE muss die Komplexität von Geschlechterverhältnissen aber konsequent auf allen Ebenen des Lehrens und Lernens von nachhaltiger Entwicklung und damit auch im GW-Unterricht mitgedacht und inkludiert werden. In dem Beitrag wird aufgezeigt, wie die Ansätze der kritischen Gender Studies den BNE-Diskurs und die Umsetzung von BNEMaßnahmen im GW-Unterricht dahingehend erweitern und die Verwendung von Stereotypen und eine Viktimisierung von Frauen verhindern können.

Keywords: Bildung für nachhaltige Entwicklung, Geschlechterverhältnis, Gerechtigkeit, Gender Studies

\section{Broadening the perspective through critical gender studies: Gender sensitive education for sustai- nable development in geography education and economic studies}

The principles of Education for Sustainable Development (ESD) are anchored in the didactic axioms and basic concepts of geography education and economic studies. The goal of ESD is to empower learners to participate in designing a sustainable and just future for all. Despite this holistic and participative approach of ESD, awareness for a gender-sensitive implementation of ESD, focusing on existing gender disparities, has so far been missing. Instead, essentialist, victimizing or gender-blind discourses dominate. Regarding the ESD's normative approach to justice, however, the complexity of gender-relations has to be kept in mind and included consistently on all levels of studying and teaching sustainable development and thus also in geography education and economic studies. This article shows how the approaches of critical gender studies can expand the ESD discourse and the implementation of ESD measures in geography and economics tuition to that effect and to avoid the use of stereotypes and the victimization of women.

Keywords: Education for Sustainable Development, gender relations, justice, gender studies

\section{$1 \quad$ Einleitung}

Die Menschheit sieht sich am Beginn des 21. Jahrhunderts mit vielfachen und komplexen Herausforderungen, wie dem globalen Klimawandel, Verlust der Biodiversität, Zerstörung von Ökosystemen,
Ressourcenverknappung, sozialen Disparitäten, gewaltvollen Auseinandersetzungen und zunehmenden Fluchtbewegungen konfrontiert. Deren Ursachen sind auf einen konsumbasierten Lebensstil und ein auf Wachstum basierendes Wirtschaftssystem zurückzuführen. Um diese Ursachen zu eliminieren, 
ist eine Veränderung unseres Verhaltens und damit auch unseres Wertedenkens erforderlich (vgl. De Haan 2008: 23 ff.; Kopfmüller et al. 2011; Stoltenberg 2009; Zimmermann 2016a: 26f.). Ziel ist es, eine gesamtgesellschaftliche Transformation zu einer nachhaltigen Entwicklung zu erreichen, „die die Bedürfnisse der Gegenwart befriedigt, ohne zu riskieren, dass künftige Generationen ihre eigenen Bedürfnisse nicht befriedigen können" (Hauff 1987). Dieser bekannten Definition einer nachhaltigen Entwicklung, welche im Brundtland-Bericht der Weltkommission für Umwelt und Entwicklung der Vereinten Nationen 1987 erstmals erschien, liegt das normative Ziel einer inter- und intragenerationellen Gerechtigkeit zu Grunde. Das bedeutet, allen Menschen - unabhängig von ihrem Geschlecht - welche sich jetzt und in $\mathrm{Zu}$ kunft auf der Erde befinden, die gleichen Möglichkeiten und Ressourcen für die Gestaltung ihres Lebens garantieren zu können. Um dies zu erreichen, ist Wissen um den Zustand unserer Welt, der bestehenden ungleichen Lebensverhältnissen von Menschen unterschiedlichen Geschlechts und ein Bewusstsein für die Notwendigkeit von Veränderung notwendig. Bildung ist hierfür der Schlüssel, der Menschen dazu befähigt, globale Zusammenhänge zu erkennen, aktuelle Praktiken zu kritisieren, Herausforderungen in ihrer ökologischen, ökonomischen und sozialen Komplexität zu verstehen, Zukunftsverantwortung wahrzunehmen und an der Gestaltung einer nachhaltigen Gesellschaft auf lokaler Ebene zu partizipieren (vgl. World Commission on Environment and Development 1987; Bundesministerium für Umwelt, Naturschutz und Reaktorsicherheit o.J.; Di Giulio 2003: 47; Michelsen \& Adomßent 2014; Zimmermann 2016b: 4; Zimmermann-Janschitz \& Wlasak 2016: 208).

Die United Nations Educational, Scientific and Cultural Organization (UNESCO) rief die Jahre 2005-2014 als Dekade für Bildung für nachhaltige Entwicklung (BNE) aus, welche das Ziel hat, „allen Menschen Bildungschancen (zu) eröffnen, die es ihnen ermöglichen, sich Wissen und Werte anzueignen sowie Verhaltensweisen und Lebensstil zu erlernen, die für eine lebenswerte Zukunft und eine positive Veränderung der Gesellschaft erforderlich sind" (UNESCO 2005: 6). BNE soll hierfür auf den formalen, non-formalen und informellen Ebenen des Lernens gefördert werdet (ebd. 2014b: 25). Nach Abschluss der Dekade wurde von den Mitgliedsstaaten der UNESCO, und damit auch von Österreich, die Umsetzung des Globalen Aktionsprogramms (GAP) für BNE als Nachfolgeinitiative der Dekade im Jahr 2015 gestartet, um das Bekenntnis zu einer umfassenden BNE zu bekräftigen und die weltweite Umsetzung von BNE weiter zu fördern (vgl. UN General Assembly 2014; UN Women 2017).
BNE ist auf österreichischer, nationaler Ebene in den didaktischen Grundsätzen des GW-Lehrplans verankert. So wird im Basiskonzept ,Nachhaltigkeit und Lebensqualität' das genannte Ziel der UNESCO direkt zitiert (Bundesministerium für Bildung 2016: 62). Was sind aber die didaktischen Grundsätze von BNE, wie werden diese im GW-Unterrichtwiedergespiegelt und berücksichtigen diese auch die unterschiedlichen und ungleichen Lebensverhältnisse von Menschen unterschiedlichen Geschlechts?

\section{Bildung für nachhaltige Entwicklung (BNE)}

Seit des Beginns der Dekade für BNE 2005 ist eine Zunahme an unterschiedlichen Definitionen von BNE zu beobachten (Bagoly-Simó 2013). Eine gemeinsame Grundlage der Herangehensweisen ist, dass BNE das Ziel hat „Menschen (zu) befähigen, sich verantwortlich und kreativ auf der Grundlage eines fundierten Wissens über komplexe Zukunftsfragen an der Gestaltung von Gegenwart und Zukunft im Sinne einer nachhaltigen Entwicklung zu beteiligen" (Stoltenberg \& Burandt 2014: 573). Genau in diesem Sinne ist auch im GW-Lehrplan verankert, dass die „Aktivität der Lernenden im Vordergrund“ stehen soll und „Unterrichtsverfahren einzusetzen“ sind, „die zu eigenständiger und kritischer Informationsverarbeitung führen. (...) Das selbstständige Erkennen von Problemen und das Finden von Wegen zu ihrer Lösung sind zu üben" (Bundesministerium für Bildung 2016: 60). Die im Lehrplan festgelegten Aspekte finden sich in weiteren pädagogisch-didaktischen Konzepten. Global Citizenship Education (GCE) bildet beispielsweise einen konzeptuellen Rahmen, in dem die Relevanz einer Bildung hervorgehoben wird, um globale Herausforderungen behandeln zu können. Sie kombiniert Theorien und Methoden der „human rights education, peace education, education for international understanding " und der Bildung für nachhaltige Entwicklung (UNESCO 2014: 9).

BNE wird als holistisches Konzept verstanden, welches für die Komplexität der Zusammenhänge ökologischer, ökonomischer und sozialer Entwicklungen auf globaler Ebene und deren Ursachen und Auswirkungen auf lokaler Ebene sensibilisiert und für konkrete Handlungen zur Sicherstellung einer intra- und intergenerationellen Gerechtigkeit anregt (vgl. De Haan 2001; Pufé 2014). Soziale Gerechtigkeit und Teilhabe von allen Menschen an einer nachhaltigen Entwicklung sind wesentliche Elemente von BNE (vgl. Embacher \& Wehling 2002).

Essentiell zur Förderung von BNE ist die Schaffung von freien Lernräumen auf allen Ebenen, in welchen Wissensaustausch, Dialog zwischen unterschiedlichen 
Akteurinnen und Akteuren aus Praxis und Wissenschaft und inter- und transdisziplinäre Lernprozesse stattfinden können, um neue Handlungsweisen zu entwickeln und erproben zu können. In diesen Lernprozessen trifft das Wissen aus unterschiedlichen Fach- und Lebenswelten aufeinander und wird basierend auf einer gegenseitigen Wertschätzung ausgetauscht und ineinander integriert (vgl. Grunwald 2002; Stoltenberg \& Burandt 2014: 579 f.). Fächerübergreifender Unterricht ist eine Möglichkeit, dies auszuüben und damit komplexe Systemzusammenhänge besser verständlich zu machen (vgl. Joppich \& Uhlenwinkel 2017). Eine solche Vorgangsweise ermöglicht eine Perspektivenerweiterung für alle Beteiligten und die Eröffnung von innovativen und alternativen Lösungsansätzen (vgl. Jahn 2008; Pohl \& Hirsch Hadorn 2006).

Weiter sind die Integration der ökologischen, ökonomischen, sozio-kulturellen und institutionellen Dimension von Nachhaltigkeit und deren Zusammenhänge essentiell, um einem ganzheitlichen Ansatz entsprechen zu können (vgl. Deller \& Spangenberg 1997; Valentin \& Spangenberg 2000). Dieses systemorientierte, vernetzte Lernen soll in einem lebensweltlichen Kontext erprobt werden und den Lernenden verdeutlichen, dass die globalen Entwicklungen Konsequenzen auf lokaler Ebene haben und sie selbst vice versa als sogenannte agents of change auf lokaler Ebene Handlungen setzen können, die die globalen Entwicklungen beeinflussen. Das Credo, Global denken - lokal handeln' soll damit unmittelbar erlebbar und anwendbar werden. Das Empowerment von unterschiedlichen Zielgruppen für konkrete Aktivitäten vor Ort ist damit immer Teil einer handlungsorientierten BNE (vgl. Michelsen \& Adomßent 2014; De Haan 2001; Huber 2001; Michelsen 2002; Stoltenberg \& Burandt 2014; Pufé 2014). Hierfür ist eine interaktive Methodenvielfalt notwendig, die Dialog, praxisorientierte Lösungsorientierung, Anerkennung von individueller Erfahrung und interdisziplinäres Wissen, aber auch kritische Reflexion inkludiert. Partizipatives und gemeinsames Lernen sollen dabei die Grundpfeiler ausmachen (vgl. Kuhn \& Heinrichs 2011).

Die Basiskonzepte des GW-Lehrplans zielen ebenso darauf $a b$, in einem ganzheitlichen Kontext „eine unübersichtliche komplexe Welt" für Schüler/ innen verständlich $\mathrm{zu}$ machen (Bundesministerium für Bildung 2016: 61), mit ihnen Orientierung, Urteils- und Handlungsfähigkeit und vernetzt und systemisch Verständnis für Komplexitäten zu entwickeln (Fridrich 2016: 24). Außerdem gilt der didaktische „Grundsatz, die Schülerinnen und Schüler zu mündiger und aktiver Partizipation im Sinne einer Bildung für nachhaltige Entwicklung zu befähigen, zu ermu- tigen und auch anzuhalten. (...) Das Unterrichtsfach Geographie und Wirtschaft leistet (...) einen Beitrag zur nachhaltigen Entwicklung sowie der Entfaltung möglichst hoher Lebensqualität für alle Menschen“" (ebd.). Die im GW-Lehrplan inkludierten entscheidungs- und handlungsleitenden Kriterien der didaktischen Grundsätze beinhalten situative und aktuelle Bezüge, exemplarische Bedeutung, Transferfähigkeit sowie fächerübergreifende Aspekte (Bundesministerium für Bildung 2016: 60). Das transdisziplinäre, alltagsweltliche und partizipative Lernen soll nach diesen Kriterien didaktisch ermöglicht werden. Die Grundelemente von BNE sind somit im Lehrplan des GW-Unterrichts verankert.

\section{Genderblindheit der BNE und des GW-Unterrichts}

BNE ist die logische Konsequenz eines transdisziplinären Verständnisses der Nachhaltigkeitswissenschaften, die an einem „gemeinsamen Lernprozess zwischen Gesellschaft und Wissenschaft anlegt und organisiert und infolgedessen reflexiv verläuft" (Michelsen \& Adomßent 2014: 41 f.). Die Basis von nachhaltiger Entwicklung und deren Bildung, eine „unerlässliche Voraussetzung für die Förderung einer nachhaltigen Entwicklung" (BMU o.J.), ist ein normativer Ansatz, der nicht nur die ökologische, sondern auch die ökonomische, kulturelle und soziale Dimension berücksichtigt und die Menschen dazu befähigt, sich mit Umwelt- und Entwicklungsfragen auseinanderzusetzen, diese abzuschätzen und Werte und Einstellungen schaffen und leben zu können (vgl. ebd.).

Nachhaltige Entwicklung, und demnach auch BNE, beinhaltet auf Basis eines schonenden wirtschaftlichen Umgangs mit bestehenden, natürlichen Ressourcen die Forderung nach sozialer Gerechtigkeit und Sicherheit der materiellen Lebensgrundlagen aller jetzt und in Zukunft lebenden Menschen in der Vielfalt ihrer Lebensrealitäten. Ein wesentliches Merkmal für soziale Gerechtigkeit ist die soziale und ökonomische Gerechtigkeit im Geschlechterverhältnis. Bestehende Geschlechterhierarchien, die Frauen strukturell, systemimmanent, direkt und indirekt mehr Arbeit, Risiken und Gewalt bei gleichzeitig weniger Kapital, Einkommen, Mitsprache, Bildungszugängen und Gesundheit aussetzen, stehen damit im Gegensatz zu einer nachhaltigen Entwicklung (vgl. Bauhardt 2010: 325). „Der Abbau der Geschlechterhierarchie ist deshalb eine notwendige Voraussetzung für die Umsetzung der Nachhaltigkeit" (ebd.) und muss auch im Rahmen von BNE thematisiert, problematisiert und gefördert werden. In Anbetracht der bestehenden weltweiten Geschlechterungleichheiten scheint dies 
offensichtlich. Beispielsweise liegt laut dem Global Gender Gap Report 2016 des World Economic Forums der Ungleichheitsfaktor von Frauen gegenüber Männern im Bereich wirtschaftlicher Chancen bei 59\%. Frauen verdienen weltweit im Durchschnitt um die Hälfte weniger als Männer obgleich sie insgesamt mehr bezahlte und unbezahlte Arbeitsstunden leisten (vgl. World Economic Forum 2016: 30; Cann 2016). Zusätzlich existieren in mehr als 150 Ländern weltweit Gesetze, die direkt Frauen diskriminieren (vgl. United Nations Development Programme 2016: 6). Auch der Zugang zu Bildung ist Mädchen eher als Burschen verwehrt: So gibt es weltweit rund 15 Millionen Mädchen im Alter zwischen 6 und 10 Jahren, welche keinen Zugang zu Schulbildung haben im Gegensatz zu rund 10 Millionen Burschen (vgl. UNESCO Institute for Statistic 2017).

Es gibt ein Bekenntnis der internationalen Gemeinschaft als auch der nationalen Regierungen, Genderungleichheiten in und durch Bildungsarbeit abzubauen (vgl. UN General Assembly 2015; UN Women 2017). Ein umfassender Ansatz, welcher eine kritische Geschlechterperspektive in Bildung für nachhaltige Entwicklung global als auch national inkludiert, fehlt aber bisher. Stattdessen scheint die Förderung und Umsetzung von BNE auf globaler als auch nationaler Ebene blind für gesellschaftliche Hierarchien zu sein, die bestehende ungleiche Geschlechterverhältnisse durch die soziale Organisation von Geschlechterordnung im jeweiligen lokalen und kulturellen Kontext reproduzieren (vgl. Wastl-Walter 2010: 198). Folgende Beispiele auf globaler und nationaler Ebene untermauern dies:

Auf globaler Ebene kommt der Dimension Geschlechtergerechtigkeit in BNE nur eine rudimentäre Rolle zu, obwohl eines der 17 Sustainable Development Goals, die 2015 von der Vereinten Nationen beschlossen wurden, explizit auf die Herstellung von Geschlechtergerechtigkeit abzielt (vgl. UN General Assembly 2015). So wird zwar in den offiziellen BNEDokumenten der UN auf die ungleiche Bildungssituation von Mädchen und Frauen weltweit aufmerksam gemacht und ein Bekenntnis zur Förderung von Mädchen und Frauen gemacht, eine konsequente Inklusion einer Gender-Perspektive fehlt hingegen. Mädchen und Frauen werden stattdessen ausschließlich in den ihnen gesellschaftlich zugeschriebenen Rolle als Mütter und Zuständige für Haushalt und Familie wahrgenommen und damit in der Privatsphäre und der Reproduktionsarbeit verortet. Zwar gibt es zahlreiche Projekte, die auf die wirtschaftliche Förderung von Frauen und damit auch ihre Rolle im öffentlichen Raum stärken (vgl. UN Women 2016), aber die UNESCO argumentiert, dass Frauen und Mädchen BNE benötigen, um sich in den Bereichen
Haushalt und Soziales an der Gestaltung einer nachhaltigen Entwicklung beteiligen können: "Vulnerable groups including girls, women, indigenous and coastal populations are hardest hit by impacts of climate change (...) and natural disasters. Especially girls and women who often play an important role in managing risks in the household and community are aimed to benefit from climate change education and education for disaster risk reduction" (UNESCO 2016). Mädchen und Frauen werden trotz ihrer vielfältigen Tätigkeiten als Familien- und Haushaltsmanagerinnen, Landarbeiterinnen, Care Workers und Arbeitnehmerinnen als verwundbare und potentielle Opfer dargestellt, die in ihren traditionell zugeordneten Arbeitsbereichen Veränderungen ermächtigt werden sollen. Diese Darstellung von Mädchen und Frauen, welche marginalisierte soziale und ökonomische Positionen in der Gesellschaft innehaben, basiert auf einer viktimisierenden Perspektive, die die Handlungsfähigkeit von Mädchen und Frauen ausschließlich in der Privatsphäre bzw. im Reproduktionsbereich sieht und dadurch unterschätzt. Mädchen und Frauen werden stattdessen als etwas Besonderes dargestellt und Stereotype (vgl. Eckes 2010), die damit verbunden sind, reproduziert und verstärkt. Diese Bezeichnung von Mädchen und Frauen als etwas Besonderes wurde bereits 1992 bei der Verabschiedung der Agenda 21 evident, in der Frauen als Hauptzielgruppe von insgesamt neun weiteren gesellschaftlichen Gruppen definiert wurden, die bei der Gestaltung einer nachhaltigen Entwicklung inkludiert werden müssen (vgl. UN General Assembly 1992; UN Department of Economic and Social Affairs 2016). Da hier Frauen als eine eigene Gruppe gesehen werden, setzt sich das Muster fort, Frauen als eine besondere Gruppe von vielen zu sehen. Mädchen und Frauen wurden demnach seit Beginn der Bestrebungen für eine nachhaltige Entwicklung nicht in ihrer Heterogenität, noch in ihrer Repräsentation der Hälfte der gesamten Menschheit gesehen.

Auf der nationalen Ebene Österreichs gilt im Schulbildungsbereich der Grundsatzerlass zum Unterrichtsprinzip ,Erziehung zur Gleichstellung von Frauen und Männern' des Bundesministeriums für Bildung. Dieser „soll dazu beitragen, alle im Bildungsbereich tätigen Personen zu motivieren, Fragen der Gleichstellung der Geschlechter verstärkt in den Lehrinhalten der Lehrpläne, im Unterricht, in den Schulbüchern und sonstigen in Verwendung stehenden Unterrichtsmitteln zu berücksichtigen sowie die Diskussion an den Schulen über diese Themen zu intensivieren" (Bundesministerium für Bildung 2014). Dies inkludiert Bewusstmachung von geschlechtsspezifischer Sozialisation, Wahrnehmung von Ursachen und Formen geschlechtsspezifischer Arbeitsteilung im Privatbereich und in der Arbeitswelt, Erkennen mög- 
licher Beiträge zur Tradierung und Verfestigung von Rollenklischees, Reflexion des eigenen Verhaltens, Bewusstmachen von Sexismus und Förderung der Bereitschaft zum Abbau von geschlechtsspezifischen Vorurteilen und Benachteiligungen (vgl. ebd.). Trotz dieses detailliert ausgeführten Prinzips, welches umfassende Dimensionen von Geschlechterungleichheit beinhaltet, und trotz des ganzheitlichen Ansatzes von BNE, der im GW-Lehrplan gefordert wird und dessen Ziel Veränderung hinsichtlich einer inter- und intragenerationellen Gerechtigkeit unabhängig von Geschlecht und jeweiliger Lebenssituation ist, wird die Dimension Geschlechtergerechtigkeit für eine nachhaltige Entwicklung im GW Lehrplan nicht explizit erwähnt. In den Basiskonzepten ,Diversität und Disparität' sowie ,Interessen, Konflikte und Macht' des GW-Lehrplan werden Hierarchien der Geschlechterverhältnisse und Ungleichheit zwischen den Geschlechtern nicht direkt genannt. Stattdessen wird darauf aufmerksam gemacht, Aspekte der Diversität und Ungleichheit zu analysieren sowie gesellschaftliche Inklusion und Exklusion und Zugang zu Ressourcen und Verteilungsfragen zu diskutieren (Bundesministerium für Bildung 2016). Es wird aber nicht darauf eingegangen, dass es sich bei diesem sozialen Phänomen um systemisch tief verwurzelte, vergeschlechtlichte Strukturen handelt, die Menschen trotz ihrer Diversität ungleiche und auf Stereotype basierende soziale Rollen und Positionen zuteilt. Auch die Vermittlung von Intersexualität und damit die Konstruktion der Vorstellung von nur zwei existierenden Geschlechtern wird nicht thematisiert (vgl. Koyama \& Weasel 2003).

Kritische Gender Studies stellen zusammengefasst fest, dass „im offiziellen Nachhaltigkeits- und Umweltbildungsdiskurs (...) Geschlechterverhältnisse und Geschlechtergerechtigkeit bislang kaum Thema" sind (Knoll \& Szalai 2005: 38). Stattdessen dominiert insbesondere auf globaler Ebene ein Viktimisierungsdiskurs, welcher Frauen vor allem „als Leidtragende der vielfältigen negativen Folgen von globalen und lokalen Umweltveränderungen betrachtet" (Bauriedl \& Wichterich 2014: 23). Es gibt Ansätze, die speziell in der Umweltpolitik der 1990er Jahre Frauen ein „geschlechtsspezifisches Expertentum für ein nachhaltiges Umweltschutzhandeln" zuschreiben und daher fordern, dass Frauen „stärker beteiligt werden, um ihre Kompetenzen als Ressourcenmanagerinnen für eine nachhaltige Entwicklung besser nutzen zu können“ (ebd.). Dieser Ansatz des Differenzfeminismus fokussiert auf die Unterschiedlichkeit von Männern und Frauen und schreibt Frauen biologisch- und sozialisationsbedingt eine Affinität und Nähe zur ,Natur' zu und schließt daraus, dass Frauen die ,besseren Umweltschützerinnen' seien. Dem setzt kritische Geschlechterforschung entgegen, dass sich Männer unter gleichen strukturellen Bedingungen gleich umweltbewusst wie Frauen verhalten (vgl. Hofmeister et al. 2013: 51). Mit der Differenzperspektive bleibt ein essentialistisch-dualistisches Geschlechterverhältnis, „das bestehende gesellschaftliche Hierarchien verschärft" bestehen (Bauriedl \& Wichterich 2014: 22 f.). So kann eine nachhaltige Entwicklung durch Bildung im ganzheitlichen und systematischen Sinne nicht gelingen, da die Ursache von Ungleichheit nämlich die Vorstellung eines nicht gleichberechtigten Verhältnisses zweier Geschlechter - nicht mitgedacht und problematisiert, sondern unbewusst reproduziert wird.

\section{Kritische Gender Studies als Perspektivenerweiterung von BNE}

Um diese Problematik zu benennen, analysieren und kritisieren zu können und schließlich zu einer Veränderung beizutragen, bedarf es einer kritischen feministischen Perspektive, welche die „Verschlechterung von sozio-ökonomischen und ökologischen Rahmenbedingungen, den für Männer und Frauen ungleichen Zugang zu Ressourcen, und die geringe Einbindung von Frauen in Entscheidungen" aufzeigt (Wastl-Walter 2010: 201). Kritische Gender Studies analysieren bestehende gesellschaftliche Verhältnisse, ihre Machtund Herrschaftsstrukturen und analysieren die soziale Konstruktion und Reproduktion von Geschlechterrollen, die daraus entstehende Chancenungleichheit und deren Effekte auf Betroffene (vgl. Aulenbacher 2010). Geschlecht wird hierbei als eine historisch sich wandelnde, gesellschaftlich kulturelle Konstruktion verstanden, welche veränderlich und veränderbar ist (vgl. Spieß 2010: 104). Gender Studies haben einen explizit herrschaftskritischen Anspruch. Sie problematisieren Machtverhältnisse, Herrschaftszusammenhänge und haben zum Ziel, die soziale Kategorie Geschlecht auf den verschiedenen (Mikro- und Makro-) Ebenen zu dekonstruieren (vgl. Franz-Balsen 2006; Hofmeister et al. 2013a). „Sie eröffnen den Blick für soziale Differenzierungen, Hierarchisierungen und Ausgrenzungen, die dann sozial-empirisch erfasst und für das Ausarbeiten der Gestaltungsperspektiven operationalisiert werden können“" (Hummel \& Schultz 2011: 230 f.). Kritische Gender Studies kritisieren den „klassischen Dualismus zwischen Natur/Frau und Kultur/ Mann“ und die „damit assoziierte Nähe der Frau zur Natur" (Wastl-Walter 2010: 85). Als problematisch wird hier gesehen, dass essentialistische und mit der Eigenschaft der ,Natürlichkeit' argumentierte Vorstellungen von weiblichen und männlichen Attributen der Aufrechterhaltung von ungleichen Herrschaftsverhältnissen dienen (vgl. ebd.; Thiessen 2010: 39 f.). 
Naturverhältnisse sind „nicht nur vergesellschaftet, sondern auch vergeschlechtlicht (...) und die Verbindung zwischen Natur, Geschlecht und Gesellschaft (...) herrschaftsförmig" (Hofmeister et al. 2013b: 78). „Ziel der feministischen Kritik ist es, zu zeigen, dass die Ausbeutung der Natur den gleichen Prinzipien folgt wie die Ausbeutung der Frau" (WastlWalter 2010: 85). Eine solche Perspektive kann aufzeigen, wie Gender als Analyse- und Untersuchungsgegenstand verwendet werden kann und Frauen aktiv in Maßnahmen für die Förderung einer nachhaltigen Entwicklung eingebunden werden sollen, um explizit Herrschaftskritik zu üben und Hierarchien zwischen den Geschlechtern aufzuzeigen (Hofmeister et al. 2013a: $46 \mathrm{f}$.).

Kritische Gender Studies gehen wie die Nachhaltigkeitswissenschaften und die genannten didaktischen Prinzipien von BNE und des GW-Unterrichts von lebensweltlichen Problemkontextualisierungen aus. Sie haben den Anspruch, durch ihre Analyse und Kritik Veränderungen herbeizuführen. Damit ist ihr Ansatz ebenso in den normativen Anspruch einer inter- und intragenerationeller Gerechtigkeit für alle Menschen, unabhängig von Geschlecht, Alter, Herkunft, Klasse und anderen sozialen Kategorien, eingebettet. Gender Studies erweitern diesen Ansatz um eine intersektionale Perspektive, welche die Wechselwirkungen zwischen unterschiedlichen sozialen Kategorien analysiert und aufzeigt, wie diese in Wechselwirkungen soziale und ökonomische Ungleichheiten in „sozialen Strukturen (d.h. von Herrschaftsverhältnissen), symbolischer Repräsentation und Identitätskonstruktionen " reproduzieren und generieren (Winkler \& Degele 2009: 15). Die intersektionale Perspektive sieht demnach nicht nur die Verstärkung von Diskriminierung und Exklusion durch das Vorhandensein von mehreren sozialen Ungleichheitskategorien, sondern erkennt und analysiert, wie deren Wechselwirkungen andere spezifische Formen von Diskriminierung hervorrufen.

Weitere Gemeinsamkeiten zwischen Gender Studies und Nachhaltigkeitswissenschaften zeigen sich dadurch, dass beide Ansätze transdisziplinär ausgerichtet sind. Beide speisen sich aus Theorien und Methoden unterschiedlicher Disziplinen und arbeiten durch ihren Anspruch, Lebensrealitäten zu verändern, transdisziplinär mit Praktikerinnen und Praktikern unterschiedlicher gesellschaftlicher Bereiche zusammen (Hofmeister et al. 2013a: $40 \mathrm{ff}$.).

Bezogen auf die Nachhaltigkeitswissenschaften und dem daraus resultierenden wissenschaftlichen Diskurs rund um BNE, der einen transdisziplinären Anspruch hat und dementsprechend das Ziel hat in der Praxis wirksam zu werden (vgl. Jahn 2008; Pohl \& Hirsch Hadorn 2006), und den Diskurs um BNE im Kontext Verankerung im Unterricht, der sich aus den transdisziplinären Inputs der Nachhaltigkeitswissenschaften ergibt, können kritische Gender Studies die Blindheit von BNE für diverse und ungleiche Lebensverhältnisse von Menschen unterschiedlichen Geschlechts aufzeigen, kritisieren wie Stereotype und daraus resultierende Ungleichheiten reproduziert werden, und damit einen Beitrag leisten, den ganzheitlichen Ansatz von BNE tatsächlich zu verwirklichen.

Basierend auf den beschriebenen Gemeinsamkeiten der beiden Ansätze besteht ein gemeinsamer Nenner, der als Türöffner fungieren kann, um die kritische Perspektive der Gender Studies im Nachhaltigkeitsdiskurs konsequent zu inkludieren. BNE ist laut Eigendefinition kritisch und partizipativ, zielt auf intra- und intergenerationelle Gerechtigkeit ab und kann es sich daher nicht leisten, eine intersektionale Gender-Perspektive auszublenden. Daher muss diese Perspektive umfassend auf allen Ebenen von BNE - sei es "Strategieebene, Akteursbene, Organisationsebene, Vermittlungsebene, Inhaltsebene" (Knoll \& Szalai 2005: 37) - inkludiert werden, um nachhaltig das Potential der Methoden einer BNE für Empowerment und Transformation nutzen zu können.

Zusammengefasst muss mit der konsequenten Inklusion einer kritischen Gender-Perspektive in BNEMaßnahmen die Verwendung von essentialistischen und stereotypischen Zuschreibungen sowie eine Viktimisierung von Frauen vermieden werden (Bauriedl \& Wichterich 2014) und damit auch ökofeministische Differenzansätze kritisiert werden. Eine kritische Gender-Perspektive ermöglicht unterschiedliche Bedürfnisse heterogener Zielgruppen wahrzunehmen, zu adressieren und zu berücksichtigen und aktive Maßnahmen gegen Ungleichheit zu setzen. Insbesondere eine intersektionale Perspektive macht es möglich, Rücksicht auf die unterschiedlichen Lebensrealitäten und ungleichen Lebensbedingungen von Personen in ihrer Vielfalt zu nehmen und diese einzubeziehen.

\section{Von der Theorie in die Praxis: Grundsätze gendersensiblen Unterrichts für die Erschließung von BNE}

Der Unterricht in der Sekundarstufe ist eine Vermittlungsebene, auf welcher die Inhalte und Kenntnisse von BNE an eine spezifische Zielgruppe, nämlich Jugendliche im Alter von 14 bis 18 Jahren, weitergegeben werden können. Gleichzeitig fordert das Bundesministerium für Bildung Schulen und Lehrer/innen auf, das Unterrichtsprinzip ,Erziehung zur Gleichstellung von Frauen und Männern' umzusetzen und sich mit Gender Mainstreaming und Geschlechtergleichstellung intensiv und reflektierend auseinanderzusetzen (vgl. Bundesministerium für Bildung 2017). 
Es gilt also BNE im Rahmen des Unterrichts gendersensibel aufzubereiten. Hierfür müssen die Grundsätze von gendersensibler Bildung eingehalten werden. Im Folgenden werden aus den Prinzipien gendersensibler Lehre zehn Grundelemente abgeleitet, die im Rahmen der Erschließung von BNE eingehalten werden sollten:

(1) Für die Gestaltung eines gendersensiblen Unterrichts ist es einerseits wichtig, Annahmen und Stereotype zu Geschlecht und Geschlechterrollen bewusst zu machen, jedoch dürfen diese dadurch nicht reproduziert oder verstärkt werden. Beispielsweise zeigen Mädchen nach wie vor in naturwissenschaftlichen und technischen Schulfächern ein überdurchschnittliches Desinteresse, das in geringen Studentinnenzahlen der entsprechenden Studienrichtungen resultiert (vgl. Amon et al. 2012: 76). Diese Problematik soll zwar angesprochen werden, jedoch in weiterer Folge sollen Beispiele gezeigt werden, wo Mädchen und Frauen nicht diesem Muster entsprechen und Freude und Erfolge in den genannten Bereichen zeigen. Es gilt einerseits die unterschiedlichen Lebenslagen und damit Lernausgangslagen der Geschlechter bewusst zu machen, ohne dabei andererseits die Unterschiede überzubetonen. Vordergründig soll in einem gender- und diversitätssensiblen Unterricht das Interesse von Kindern und Jugendlichen unabhängig von Geschlecht gefördert werden und dabei Stereotype durchbrochen werden (vgl. Amon et al. 2012: 76). „Geschlechterorientierung, die auf die Entwicklung der Potenziale beider Geschlechter zielt, muss von Fach zu Fach, von Lernbereich zu Lernbereich geschlechtersensibel und differenziert ausgestaltet werden, um Geschlechterstereotypisierung langfristig aufzubrechen" (Hoppe \& Nyssen 2004: 239). Werden beispielsweise wirtschaftliche Bedürfnisse der Menschen im Rahmen des GW-Unterrichts bewertet (vgl. Bundesministerium für Bildung 2016: 64), so sollen hierbei unterschiedliche Bedürfnisse von Frauen und Männern thematisiert werde, die sozio-ökonomischen Gründe hierfür aber erklärt werden und Gründe, die auf Stereotypen beruhen, bewusst problematisiert werden. Eine Möglichkeit hierfür stellt das Kennenlernen von alternativen Lebensweisen, die duale Geschlechterrollen nicht reproduzieren, an Hand von Fallbeispielen dar.

Um dies tun zu können, ist ein (2) grundlegendes Wissen der Lehrenden zur sozialen Konstruktion von Geschlecht notwendig. Gender-Kompetenz sollte bei Lehrenden vorhanden sein und gefördert werden. Denn Lehrer/innen müssen dafür sensibilisiert sein, dass Geschlecht eine hierarchisch verfasste Machtund Strukturkategorie ist, die historisch und kulturell variabel, daher nicht konstant ist und nicht die Eigenschaft einer Person bestimmt. Auf individueller Ebene bedeutet dies auch, dass Lehrende persönlich ihre eigenen Normen und Werte, „die im Laufe der biographischer Begegnungen konstruiert wurden, (...) reflektieren und (...) dekonstruieren und diese somit geschlechtssensibel und auf persönlicher Ebene zu bearbeiten" (Bundesministerium für Unterricht, Kunst und Kultur 2009: 8f.). Dementsprechende Fort- und Weiterbildungen in Form von qualitativ-hochwertigen Gender-Trainings, aktive selbstständige Reflexion und gegenseitiges Feedback sind hierfür notwendig.

Auf Basis dieser Gender-Kompetenz gilt es den Unterricht hinsichtlich seiner pädagogischen didaktischen und inhaltlichen Ausrichtung so zu gestalten, dass (3) die Geschlechterperspektive durchgehend mitgedacht und reflektiert wird (vgl. Bundesministerium für Bildung 2017).

Eine solche Herangehensweise macht es möglich, dass Schüler/innen in ihrem Lernen nicht in Geschlechterrollen gedrängt werden oder darin ausharren, sondern dass sie (4) nach individuellen Interessen, Fähigkeiten, Fertigkeiten sowie sozialen und individuellen Ausgangslagen gleichermaßen angesprochen werden. Es gilt zu vermeiden Mädchen und Burschen unterschiedlich wahrzunehmen sowie bewusst und unbewusst unterschiedliche Erwartungen an sie $\mathrm{zu}$ stellen (vgl. Bundesministerium für Unterricht, Kunst und Kultur 2009). Dies schränkt die Schüler/ innen in ihrem Potential ein und drängt sie in Stereotype und Rollenmuster, die es aber gilt aufzubrechen.

Dazu sollen Lehrende (5) ein vertrauensvolles, sicheres Lernklima schaffen, das alle Schüler/innen dazu ermutigt, sich unter Berücksichtigung ihrer Lernbedürfnisse einzubringen. Die hierfür notwendigen Regeln für respektvollen Umgangs- und Kommunikationsformen werden im Vorfeld mit der gesamten Klasse gemeinsam erarbeitet. Es gilt (6), Mädchen und Burschen in einem geschützten und barrierefreien Raum anzuregen, auf gleiche Weise aktiv mitzuwirken und sie in ihrem Selbstbewusstsein und ihren individuellen Interessen zu stärken. Den Schülerinnen und Schülern wird klar kommuniziert, dass die Beiträge und Fragen aller erwünscht und wertgeschätzt werden. Dies kann je nach Zielen des Unterrichts entweder ko- oder monoedukativ erfolgen. Wesentlich ist, dass sich alle Schüler/innen frei fühlen, ihre Fragen und ihr Wissen einzubringen. Die (7) Grundwerte eines gleichberechtigten, wertschätzenden Umgangs miteinander müssen hierfür klar adressiert und (selbst-)reflektierend mit den Schülerinnen und Schülern besprochen werden. Außerdem müssen Alternativen und neue Perspektiven außerhalb von heteronormativen, traditionellen Geschlechterbias aufgezeigt werden (Mörth 2010: 67f.), um mögliche Handlungsspielräume zu erweitern.

Ein Kernelement bildet (8) die Sprache als Schlüssel zur Öffnung dieser Handlungsspielräume. Sprache 
konstruiert Wirklichkeit und kann, wenn reflektiert, zur Veränderung des Denkens und der Wirklichkeit führen (Mörth 2010: 68; Spieß 2010: 105f.). Dies inkludiert die klare und durchgehende Benennung aller Geschlechter in Wort und Schrift und das bewusste Vermeiden von stereotypen Beispielen und Darstellungen, aber auch die bewusste Wahl einer gendersensiblen Sprache (vgl. Bundesministerium für Unterricht, Kunst und Kultur 2008).

Im Unterricht verwendete Lehrmaterialien müssen (9) ebenso in diesem Sinne durch verschiedenste Medien aufbereitet sein und Geschlechter gleichermaßen und doch in ihrer Vielfalt und in ihren ungleichen Lebensbedingungen visuell und sprachlich repräsentieren (Spieß 2010: 107), ohne hierbei einen Fokus auf Differenz zu legen (vgl. Soiland 2009). Bei der Verwendung von verschiedensten Medien ist es dabei wichtig, den Mainstream dominierende Stereotypen kritisch zu hinterfragen, welche weibliches und männliches Rollenverhalten reproduzieren und verstärken (vgl. Angerer \& Dorer 1994).

Wesentlich ist, dass der Ansatz eines gendersensiblen Lehren und Lernens (10) ganzheitlich institutionalisiert und demnach auch im Lehrplan, in den Curricula, in der Aus- und Fortbildung der Lehrenden und in bildungspolitischen Strategien zum Ausgleich von bestehenden Ungleichheiten und bei Bedarf in der spezifischen Mädchen- und Burschenförderung verankert ist (vgl. Schrodt 2010).

Wir sehen also, dass die didaktischen Prinzipien einerseits auf die unterschiedlichen Bedürfnisse von Mädchen und Jungen eingehen, wir jedoch gleichzeitig stets einen kritischen Blick auf vermeintliche Essentialisierung und Stereotypisierung werfen müssen. Es gilt Geschlechterungleichheiten anzusprechen, ohne sie zu naturalisieren oder zu reproduzieren. Insbesondere letzteres lässt sich durch konsequente aktive Einholung von alternativen Handlungsmustern vermeiden.

\section{$6 \quad$ Fazit}

Das holistische Konzept von BNE benötigt eine kritische Gender-Perspektive, um bestehende Ungleichheiten und Diskriminierungen zwischen den Geschlechtern zu erkennen, diese in ihren Ursprüngen kritisch zu analysieren und Maßnahmen dagegen auf allen Bildungsebenen zu ergreifen. Um alle Menschen an der Gestaltung einer inter- und intragenerationellen Weltgesellschaft teilhaben zu lassen, ist eine kritische Gender-Perspektive essentiell, um Essentialismen, Reproduktion von Stereotypen und Viktimisierungen zu vermeiden. Die Ansätze einer kritischen GenderPerspektive zeigen zahlreiche Gemeinsamkeiten mit den ebenso normativen Ansprüchen der Nachhaltigkeitsforschung auf. So haben beide zum Ziel, sich auf transdisziplinäre Weise an den Bedürfnissen und Lebensrealitäten heterogener Zielgruppen zu orientieren und partizipativ Veränderungen an bestehenden Verhältnissen, die soziale, ökologische und ökonomische Ungleichheiten verursachen, zu bewirken.

Um einen konkreten Beitrag für die Beseitigung von Geschlechterungleichheit im Kontext nachhaltiger Entwicklung zu leisten, müssen Lehrer/innen dazu aufgefordert und ermutigt werden, (1) BNE im Sinne eines fächerübergreifendenden, inter- und transdisziplinären GW-Unterrichts, der die Lebensrealitäten der Schüler/innen betrifft, weiter zu verankern und (2) darin eine konsequente kritische GenderPerspektive zu verfolgen.

Das Ziel hiervon ist es, einen gendersensiblen Unterricht zu realisieren, in welchem die Schüler/innen ermutigt werden, sich mit Themen der Gestaltung einer nachhaltigen Zukunft im Kontext ihrer jeweiligen, diversen Lebensrealitäten auseinanderzusetzen und hierbei ein Bewusstsein dafür zu entwickeln, in welchen dieser Lebensrealitäten und gesellschaftlichen Strukturen Geschlechterungleichheiten vorkommen und wie Verbesserungen bzw. Alternativmodelle hierzu aussehen könnten. Genau dies entspricht auch dem Ziel des GW-Unterrichts, welches mit dem Kompetenzmodell, dem neuen Lehrplan und insbesondere den Basiskonzepten verankert ist, Interdependenzen menschlichen Handelns zu erkennen und sich selbst aktiv an der Gestaltung einer nachhaltigen Gesellschaft und Zukunft zu beteiligen. In diesem Kontext gilt es, durch die Inklusion einer kritischen GenderPerspektive, die gesellschaftliche Verankerung von Geschlechterungleichheit und deren negative Konsequenzen zu thematisieren und zu problematisieren. Die didaktischen Methoden für die Umsetzung eines gendersensiblen Unterrichts sind vorhanden; nun gilt es auf struktureller Ebene gendersensiblen Unterricht mit den Aufgaben des GW-Unterrichts für die Förderung einer BNE zu koppeln und nicht wie bisher, als zwei parallel bestehende Grundsätze zu behandeln. Im beiliegenden Unterrichtspraxis-Beitrag, Praktische Umsetzung von gendersensibler Bildung für nachhaltige Entwicklung im GW-Unterricht' wird ein konkretes Stundenbild, wie dies gelingen kann, vorgestellt.

\section{Dank und Förderhinweis}

\section{Publiziert mit Unterstützung der Universität Graz}

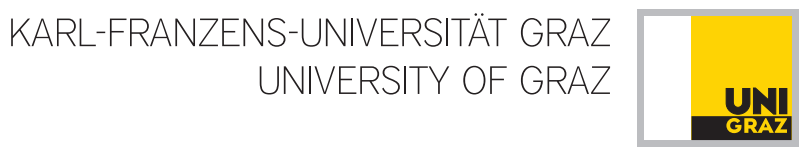




\section{$7 \quad$ Literatur}

Amon,H., I. Bartosch, A. Lembens \&I. Wenzl (2012): Gender-Diversity-Kompetenz im naturwissenschaftlichen Unterricht. Fachdidaktische Anregungen für Lehrerinnen und Lehrer. IMST (Innovationen Machen Schulen Top) - Gender Diversitäten Netzwerk (Hrsg.). Wien.

Angerer, M.-L. \& J. Dorer (1994): Gender und Medien: Theoretische Ansätze, empirische Befunde und Praxis der Massenkommunikation. Ein Textbuch zur Einführung. Wien: Wilhelm Braumüller Verlag.

Aulenbacher, B. (2010): Gesellschaftsanalysen der Geschlechterforschung. In: ebenda, M. Meuser \& B. Riegraf (Hrsg.): Soziologische Geschlechterforschung. Eine Einführung. Wiesbaden: VS Verlag, 15-33.

Bagoly-Simó, P. (2013): Tracing Sustainability: An International Comparison of ESD Implementation into Lower Secondary Education. In: Journal of Education for Sustainable Development 7/1, 95-112.

Bauhardt, C. (2010): Ökologiekritik. Das Mensch-NaturVerhältnis aus der Geschlechterperspektive. In: Becker, R. \& B. Kortendiek (Hrsg.): Handbuch Frauen- und Geschlechterforschung. Theorie, Methoden, Empirie. 3. erweiterte und durchgesehene Auflage. Wiesbaden: VS Verlag, 322-327.

Bauriedl, S. \& C. Wichterich (2014): Ökonomisierung von Natur, Raum, Körper. Feministische Perspektiven auf sozialökologische Transformationen. Berlin: RosaLuxemburg-Stiftung. https://www.rosalux.de/fileadmin/ rls_uploads/pdfs/Analysen/Analysen_Oekonomisierung. $\operatorname{pdf}(23.10 .2017)$.

Bundesministerium für Bildung (2014): Grundsatzerlass zum Unterrichtsprinzip „Erziehung zur Gleichstellung von Frauen und Männern“. Geschäftszahl: 15.510/60Präs.3/95. Wien. https://www.bmb.gv.at/ministerium/ rs/1995_77.html (23.10.2017).

Bundesministerium für Bildung (2016): Bundesgesetzblatt für die Republik Österreich. 219. Änderung der Verordnung über die Lehrpläne der allgemein bildenden höheren Schulen; Änderung der Bekanntmachung der Lehrpläne für den Religionsunterricht an diesen Schulen. Teil II. Wien. https://www.ris.bka.gv.at/Dokumente/BgblAuth/BGBLA_2016_II_219/BGBLA_2016_II_219. pdf (23.10.2017).

Bundesministerium für Bildung (2017): Erziehung zur Gleichstellung von Frauen und Männern, Wien. https:// www.bmb.gv.at/schulen/unterricht/prinz/erziehung_ gleichstellung.html (23.10.2017).

BMU: Bundesministerium für Umwelt, Naturschutz und Reaktorsicherheit (o. J.): Konferenz der Vereinten Nationen für Umwelt und Entwicklung im Juni 1992 in Rio de Janeiro; Agenda 21. http:/www.bmu.de/files/pdfs/ allgemein/application/pdf/agenda21.pdf (23.10.2017).

Bundesministerium für Unterricht, Kunst und Kultur (2008): Gender Kompetenz und Gender Mainstreaming. Kriterienkatalog für Schulen. Wien. http://www.eduhi. at/dl/gekos-kriterien-online.pdf(23.10.2017).
Bundesministerium für Unterricht, Kunst und Kultur (2009): Geschlechtssensible Pädagogik. Leitfaden für Lehrer/innen und Fortbildner/innen im Bereich Kindergartenpädagogik. Wien. Pubshop.bmbf.gv.at/download. aspx?id=184 (23.10.2017).

Cann, O. (2016): Gender equality is sliding backwards, finds our global report. https://www.weforum.org/ agenda/2016/10/gender-gap-report-2016-equalitysliding-backwards?utm_content=buffer698c6\&utm_ medium=social $\&$ utm_source=twitter. $\operatorname{com} \& u$ tm campaign=buffer $(23.10 .2017)$.

De Haan, G. (2001): Was meint „Bildung für nachhaltige Entwicklung" und was können eine globale Perspektive und neue Kommunikationsmöglichkeiten zur Weiterentwicklung beitragen? In: Herz, O., H. Seybold \& G. Strobl (Hrsg.): Bildung für nachhaltige Entwicklung. Globale Perspektiven und neue Kommunikationsmedien. Opladen: Leske und Budrich, 29-45.

De Haan, G. (2008): Gestaltungskompetenz als Kompetenzkonzept der Bildung für nachhaltige Entwicklung. In: Bormann, I. \& G., de Haan (Hrsg.): Kompetenzen der Bildung für nachhaltige Entwicklung. Operationalisierung, Messung, Rahmenbedingungen, Befunde. Wiesbaden: VS Verlag für Sozialwissenschaften, 23-43

Deller, K. \& J. Spangenberg (1997): Fünf Jahre nach dem Erdgipfel. Wie zukunftsfähig ist Deutschland? Entwurf eines alternativen Indikatorensystems. Forum Umwelt und Entwicklung, Bonn.

Di Giulio, A. (2003): Die Idee der Nachhaltigkeit im Verständnis der Vereinten Nationen. Anspruch, Bedeutung und Schwierigkeiten. Münster: LIT.

Eckes, T. (2010): Geschlechterstereotype: Von Rollen, Identitäten und Vorurteilen. In: Becker, R. \& B. Kortendiek (Hrsg.): Handbuch Frauen- und Geschlechterforschung. Theorie, Methoden, Empirie. 3., erweiterte und durchgesehene Auflage. Wiesbaden: VS Verlag, 178-189.

Embacher, C. \& P. Wehling (2002): Soziale Dimensionen der Nachhaltigkeit. Theoretische Grundlagen und Indikatoren. Studientexte des Instituts für sozial-ökologische Forschung 11. Frankfurt am Main.

Franz-Balsen, A. (2006): Genderforschung - Herausforderung für die Bildung für nachhaltige Entwicklung. In: Rieß W. \& H. Apel (Hrsg.): Bildung für eine nachhaltige Entwicklung. Wiesbaden: Springer, 185-195.

Fridrich, C. (2016): Basiskonzepte in Geographie und Wirtschaftskunde - ein Vorschlag für die Sekundarstufe I. In: GEOGRAZ. Grazer Mitteilungen der Geographie und Raumforschung 59, 24-31.

Grunwald, A. (2002): Technikfolgenabschätzung. Eine Einführung. Gesellschaft - Technik - Umwelt, Band 1. Berlin: Edition Sigma.

Hauff, V. (1987): Unsere gemeinsame Zukunft. Der Brundtland-Bericht der Weltkommission für Umwelt und Entwicklung. Eggenkamp: Greven.

Hofmeister S., C. Katz \& T. Mölders (2013a): Grundlegungen im Themenfeld Geschlechterverhältnisse und 
Nachhaltigkeit. In: ebd. (Hrsg.): Geschlechterverhältnisse und Nachhaltigkeit. Die Kategorie in den Nachhaltigkeitswissenschaften. Opladen, Berlin, Toronto: Barbara Budrich, 31-76.

Hofmeister S., C. Katz \& T. Mölders (2013b): Orientierung im Themenfeld Geschlechterverhältnisse und Nachhaltigkeit. In: ebd. (Hrsg.): Geschlechterverhältnisse und Nachhaltigkeit. Die Kategorie in den Nachhaltigkeitswissenschaften. Opladen, Berlin, Toronto: Barbara Budrich, 77-150.

Hoppe, H. \& E. Nyssen, (2004): Gender Mainstreaming: Neue Gleichstellungsimpulse für die Schule? Begründungen und Ansatzpunkte. In: Meuser, M. \& C. Neusüß (2004): Gender Mainstreaming. Konzepte - Handlungsfelder - Instrumente. Berlin: Bundeszentrale für Politische Bildung, 232-243.

Huber, L. (2001): Anfragen an das Konzept einer BNE. In: Herz O., H. Seybold \& G. Strobl (Hrsg.): Bildung für nachhaltige Entwicklung. Globale Perspektiven und neue Kommunikationsmedien. Opladen: Lekse und Budrich, 77-86.

Hummel, D. \& I. Schultz (2011): Geschlechterverhältnisse und gesellschaftliche Naturverhältnisse - Perspektiven Sozialer Ökologie in der transdisziplinären Wissensproduktion. In: Scheich E. \& K. Wagels (Hrsg.): Körper Raum Transformation. Gender-Dimensionen von Natur und Materie. Forum Frauen- und Geschlechterforschung, Band 32, Münster: Westfälisches Dampfboot, 218-233.

Jahn, T. (2008): Transdisziplinarität in der Forschungspraxis. In: Bergmann, M. \& E. Schramm (Hrsg.): Transdisziplinäre Forschung. Integrative Forschungsprozesse verstehen und bewerten. Frankfurt/Main, New York: Campus Verlag, 21-37.

Joppich, A. \& A. Uhlenwinkel (2017): Fächerübergreifender Unterricht zur Nachhaltigkeit: wissens- oder verhaltensorientiert? In: GW-Unterricht 145, 18-27. http://www.gw-unterricht.at/index.php/19-onlineausgaben/2017/54-145-2017.html (23.10.2017).

Knoll, B. \& E. Szalai (2005): Gender und Bildung für Nachhaltige Entwicklung. Im Auftrag des Bundesministerium für Land- und Forstwirtschaft, Umwelt und Wasserwirtschaft, Wien. http://www.b-nk.at/wp-content/ uploads/2015/07/B-NK-2005-Gender_BildungfuernachhaltigeEntwicklung_Broschuere.pdf (23.10.2017).

Kopfmüller, J., V. Brandl, J. Jörissen, M. Paetau, G. Banse, R. Coenen \& A. Grunwald (2011): Nachhaltige Entwicklung integrativ betrachtet. Konstitutive Elemente, Regeln, Indikatoren. Berlin: Sigma.

Koyama, E. \& L. Weasel (2003): From Social Construction to Scoial Justice. Transformierung How We Teach About Intersexuality. In: Intersex Initiative Portland (Hrsg.): Teaching Intersex Issues. A Guide for Teachers in Women's, Gender \& Queer Studies. The Second Edition. Portland Oregon. http://eminism.org/store/pdf-zn/ ipdx-teacher.pdf (23.10.2017).

Kuhn, K. \& H. Heinrichs (2011): Partizipation, Kooperation und nachhaltige Entwicklung im Kontext globalen
Wandels. In: Heinrichs, H., K. Kuhn \& J. Newig (Hrsg.): Nachhaltige Gesellschaft. Welche Rolle für Partizipation und Kooperation? Wiesbaden: Springer, 15-24.

Michelsen, G. (2002): Bildung und Kommunikation für eine Nachhaltige Entwicklung: Sozialwissenschaftliche Perspektiven. In: Beyer, A. (Hrsg.): Fit für Nachhaltigkeit? Biologisch-anthropologische Grundlagen einer Bildung für nachhaltige Entwicklung. Wiesbaden: VS Verlag für Sozialwissenschaften, 193-216.

Michelsen, G. \& M. Adomßent (2014): Nachhaltige Entwicklung: Hintergründe und Zusammenhänge. In: Heinrichs, H. \& G. Michelsen (Hrsg.): Nachhaltigkeitswissenschaften. Berlin, Heidelberg: Springer, 3-59.

Mörth, A. (2010): Handlungsvorschläge für einen nicht-binären Umgang mit Geschlecht. In: Mörth, A. \& B. Hey (Hrsg.): Geschlecht und Didaktik. 2. überarbeitete Auflage. Graz: Grazer Universitätsverlag, 61-70.

Pohl, C. \& G. Hirsch Hadorn (2006): Gestaltungsprinzipien für die transdisziplinäre Forschung. München: oekom.

Pufé, I. (2014): Was ist Nachhaltigkeit? Dimensionen und Chancen. In: Aus Politik und Zeitgeschichte (APuZ): Nachhaltigkeit 64, 15-21. http://www.bpb.de/ apuz/188663/was-ist-nachhaltigkeit-dimensionen-undchancen?p=all (23.10.2017).

Schrodt, H. (2010): Mädchenförderung? Bubenförderung? Gender Mainstreaming? Auf dem Weg zu einer geschlechtssensiblen Schule. In: Mörth, A. \& B. Hey (Hrsg.): Geschlecht und Didaktik. 2. überarbeitete Auflage, Graz: Grazer Universitätsverlag, 31-40.

Soiland, T. (2009): Gender als Selbstmanagement. Zur Reprivatisierung des Geschlechts in der gegenwärtigen Gleichstellungspolitik. In: Andresen S., M. Korebuer \& D. Lüdke (Hrsg.): Gender und Diversity: Albtraum oder Traumpaar? Interdisziplinärer Dialog zur „Modernisierung" von Geschlechter- und Gleichstellungspolitik. Wiesbaden: VS Verlag, 35-52.

Spieß, G. (2010): Voll gesellschaftsfähig! - mit einer gendersensiblen Lehre. Eine Materialsammlung. In: Mörth, A. $\&$ B. Hey (Hrsg.): Geschlecht und Didaktik. 2. überarbeitete Auflage. Graz: Grazer Universitätsverlag, 95-134.

Stoltenberg, U. (2009): Mensch und Wald. Theorie und Praxis einer Bildung für eine nachhaltige Entwicklung am Beispiel des Themenfelds Wald. München: Oekom.

Stoltenberg, U. \& S. Burandt (2014): Bildung für eine nachhaltige Entwicklung. In Heinrichs, H. \& G. Michelsen (Hrsg.): Nachhaltigkeitswissenschaften. Berlin, Heidelberg: Springer Spektrum, 567-594.

Thiessen, B. (2008): Feminismus: Differenzen und Kontroversen. In: Becker, R. \& B. Kortendiek (Hrsg.): Handbuch Frauen- und Geschlechterforschung. Theorie, Methoden, Empirie. 3. erweiterte und durchgesehene Auflage, Wiesbaden: VS Verlag, 37-44.

UN Department of Economic and Soical Affairs (2016): Sustainable Development Platform. About Major Groups and other stakeholders. https://sustainabledevelopment. un.org/aboutmajorgroups.html(23.10.2017). 
UN General Assembly (1992): Report of the United Nations Conference on Environment and Development, Rio de Janeiro, 3-14 June 1992. http://www.un.org/documents/ga/conf151/aconf15126-3.htm(23.10.2017).

UN General Assembly (2014): Resolution adopted by the General Assembly on 19 December 2014. Follow-up to the United Nations Decade of Education for Sustainable Development (2005-2014): Global Action Programme on Education for Sustainable Development http://www. unesco.at/bildung/gap_res_n1471200.pdf(23.10.2017).

UN General Assembly (2015): Resolution adopted by the General Assembly on 25 September 2015. Transforming our world: the 2030 Agenda for Sustainable Development. http://www.un.org/ga/search/view_doc. asp?symbol=A/RES/70/1 \&Lang=E(23.10.2017).

UNESCO (2005): United Nations Decade of Education for Sustainable Development (2005-2014). International Implementation Scheme. https://www.bibb.de/dokumente/pdf/a33_unesco_international_implementation_scheme.pdf (23.10.2017).

UNESCO (2014a): Global Citizenship Education. Preparing learners for the challenges of the 21 st century. http:// unesdoc.unesco.org/images/0022/002277/227729e.pdf (23.10.2017).

UNESCO (2014b): Roadmap for Implementing the Global Action Programme on Education for Sustainable Development. http://unesdoc.unesco.org/ images/0023/002305/230514e.pdf (23.10.2017). (Deutschsprachige Fassung: http://www.bne-portal.de/ sites/default/files/_2015_Roadmap_deutsch_0.pdf).

UNESCO (2016): Gender Equality in Education. http:// www.unesco.org/new/en/education/themes/leading-theinternational-agenda/education-for-sustainable-development/gender-equality/ (23.10.2017).

UIS: UNESCO Institute for Statistic (2017): UNESCO eAtlas of Gender Inequality in Education. http://tellmaps. com/uis/gender/\#!/tellmap/79054752 (23.10.2017).

United Nations Development Programme (2016): Human Development Report 2016. Human Development for Everyone. http://hdr.undp.org/sites/default/files/2016_ human_development_report.pdf (23.10.2017).
UN Women: United Nations Entity for Gender Equality and the Empowerment of Women (2016): Empowering women farmers of Rwanda through mobile technology. http://www.unwomen.org/en/news/stories/2016/10/empowering-women-farmers-of-rwanda-through-mobiletechnology (23.10.2017).

UN Women: United Nations Entity for Gender Equality and the Empowerment of Women (2017): The Beijing Platform for Action: inspiration then and now. http:// beijing20.unwomen.org/en/about (23.10.2017).

Valentin, A. \& J.H, Spangenberg (2000): A guide to community sustainability indicators. In: Environmental Impact Assessment Review 20/3, 381-392.

Wastl-Walter, D. (2010): Gender Geographien. Geschlecht und Raum als soziale Konstruktion. Sozialgeographie kompakt, Band 2. Stuttgart: Franz Steiner Verlag.

Winkler G. \& N. Degele (2009): Intersektionalität. Zur Analyse sozialer Ungleichheiten. Bielefeld: transcript.

WCED: World Commission on Environment and Development (1987): Our Common Future (Unsere gemeinsame Zukunft) = Brundtland-Bericht. http://www.undocuments.net/wced-ocf.htm (23.10.2017).

World Economic Forum (2016): The Global Gender Gap Report 2016. http://www3.weforum.org/docs/ GGGR16/WEF_Global_Gender_Gap_Report_2016. pdf (23.10.2017).

Zimmermann, F. M. (2016a): Globale Herausforderungen und die Notwendigkeit umzudenken - wie soll das funktionieren? In: ebd. (Hrsg.): Nachhaltigkeit wofür? Von Chancen und Herausforderungen für eine nachhaltige Zukunft. Berlin, Heidelberg: Springer, 25-58.

Zimmermann, F. M. (2016b): Was ist Nachhaltigkeit eine Perspektivenfrage? In: ebd. (Hrsg.): Nachhaltigkeit wofür? Von Chancen und Herausforderungen für eine nachhaltige Zukunft. Berlin, Heidelberg: Springer, 1-24.

Zimmermann-Janschitz, S. \& P. Wlasak (2016): Nachhaltigkeit, Gerechtigkeit und Inklusion - Zukunftskonzept oder Wunschtraum? In: Zimmermann, F. M. (Hrsg.): Nachhaltigkeit wofür? Von Chancen und Herausforderungen für eine nachhaltige Zukunft. Berlin, Heidelberg: Springer, 201-228. 